\title{
BAI-Net: Individualized Anatomical Cerebral Cartography using Graph Convolutional Network
}

\author{
Liang Ma, Yu Zhang, Hantian Zhang, Luqi Cheng, Junjie Zhuo, Weiyang Shi, Yuheng Lu, Wen Li, \\ Zhengyi Yang, Jiaojian Wang, Lingzhong Fan and Tianzi Jiang, Fellow, IEEE
}

\begin{abstract}
Individualized human cerebral cartography has drawn considerable attention in neuroscience research. Many challenges remain, mainly due to large individual variability in brain morphology, connectivity and function. We developed a new tool called brain atlas individualization network (BAl-Net) that automatically parcels individual cerebral cortex into segregated areas using structural and diffusion MRIs. The presented method integrates the group priors into the loss function of graph convolution network, learns the connectivity context of anatomical fingerprints for each area, and consequently provides reliable and explainable individual-specific topography across multiple sessions and different scanners. The BAl-Net model not only captures high variations across individual brains while maintaining high consistency with the priors in the group level, demonstrating high heritability in a twin study, but also shows high associations with behaviors in a cognitive battery. Our method shows high potentials in the field of precision medicine including the diagnosis and treatment of neurological disorders.
\end{abstract}

Index Terms - cerebral cartography, individual parcellations, functional alignment, anatomical fingerprints, graph convolutional network, heritability

\section{INTRODUCTION}

$\mathrm{T}$ he human cerebral cortex can be divided into multiple function-specific areas in terms of distinct in vivo and ex vivo features. To understand the functional organization of the human brain, neuroanatomists have built a variety of brain atlases to depict macro- and microanatomical architectures (e.g., cytoarchitecture and the distribution of neuroreceptors and myelination) using a few postmortem human brains [1-7]. As the technology advances, magnetic resonance imaging (MRI) techniques, have been used to investigate in vivo brain features (e.g., macrostructure, connectivity, and functional activations) as well as to make subsequent cartographic explorations of the human cerebral cortex at the population level [8-16]. However, many studies presented the individual variations of cerebral cortex including areal size, location, spatial arrangement and connectivity patterns, as a result of genetic and environmental influences $[15,17]$. Individual-specific topography of cerebral cartography would improve cross-subject analysis and reveal behavior-related, disease-dependent locations for personalized medical practice [18-24].

In-vivo cortical fingerprint-based (e.g., anatomical and functional connectivity) mappings are adopted to reveal the areal individual differences [13, 25-33]. Among these mappings, group atlas priors provide a blueprint of general architectures of the human cerebral cortex to guide the individual-specific cartography [13, 23, 26, 32-37]. Resting-state functional MRI (rs-fMRI) individualized methods have been first introduced and made well applications in analyzing the individual brain functions and related behaviors and mental illnesses [37, 38]. Anatomical fingerprints (e.g., macroanatomical strcuture of brain cortex) derived from diffusion MRI are also substantial indicators to reflect individual variations of cerebral cortex. Previous efforts have shown that there exists a high overlap between tractography-based cerebral cartography using diffusion MRI (dMRI) with cytoarchitectonic areas [39]. It would help to unravel the individual anatomical differences based on the individual anatomical cerebral cartography.

Current efforts to achieve anatomical-based individualized methods are so far relied on the adoption of local microstructures and original fiber tractography [30, 40]. And many of them to achieve an individual-specific parcellation followed the way of rs-fMRI methods. For example, Han et. al. (2019) has brought an anatomical individualization method (denoted as iterative clustering (IC) method) which are originally applied in the rs-fMRI [30]. However, due to the differences among two modalities, there still exists a great challenge for achieving a robust, individual-specific topography of cerebral cortex in anatomy that can reveal individual differences in cognition and behaviors [41]. First of all, unlike functional connectivity, it exists in a fiber tracking that the observed frequency of connection is dominated simply by distance effects [42]. Direct utilization of original fiber tractography not only lacks of interpretations but also puts a risk to reflect the individual idiosyncrasies. Second, the integration of population priors in the individualized method should effectively constrain the anatomical connectivity for the crosssubject analysis. Previous method may have less restrictions on the population consistency of the areal connectivity across subjects, which may put an obstacle on the cross-subject analysis. Finally, there might lacks multi-range references to enhance the reliability of areal delineation. The integration of the multi-range connectivity references are always reliable than the single one.

When faced with these problems, machine learning methods provide alternative solutions with their flexible modeling, powerful data fitting, and inference capabilities. First of all, fiber-tract-based fingerprints have been applied in the location of homological areas in the cross-species comparison [43]. Such fingerprints could also reflect a reliable and explainable variations for homological areas across subjects. Precise fiber tracts of an individual brain, the necessities of connectivity fingerprints, can be segmented automatedly by machine 

available under aCC-BY-NC-ND 4.0 International license.

learning methods[44]. Second, the group priors could be directly constrained by the distribution of areal connectivity. In other words, the anatomical connectivity of each area follows a certain distribution on a large population, which can be learnt by a machine learning method. Third, the spatially connectivity information from neighborhood vertexes (connectivity context) could provide alternative landmarks to enhance the area identification, since spatial distribution of connectivity on cerebral cortex have the spatial intrinsic associations. Graph convolutional networks make this context-based analysis possible on non-Euclidian geometric space (e.g., the brain cortical surface) using a few graph convolutional kernels (e.g., Chebyshev polynomials). It is also convenient to quickly inference a new data using a trained model in a practical application.

With the integration of these advantages, we developed a Brain Atlas Individualization Network (BAI-Net) using group priors (in here, we use the Human Brainnetome Atlas [14] in the experiment). The presented study introduces a graph-based individualization method for reliable individual-specific parcellation. It improved the cross-subject, atlas-based analysis and might assistant precise locations in many illness treatments (e.g., transcranial magnetic stimulation). The advantages of the presented model are shown below:

1) First, we used the individual-level anatomical connectivity fingerprint derived from fiber-tract embedding of the diffusion tractography to handle the anatomical connectivity complexity as well as to provide the model interpretability. The resulting individual parcellations showed a high reproducibility, large individual variations.

2) Second, the connectivity context is introduced for reliable areal identification using graph convolution networks. A connectivity-based brain graph (spatial structure and fingerprints) was first constructed for individualization methods. Probabilistic weights and population locations were integrated into the loss function to search a reliable connectivity context pattens for cortical areas. The high population consistency and reliable inter-subject variations of the individual-specific topography of cerebral cartography shows an improved association with 58 individual cognition and behaviors as well as higher heritability than the IC method.

\section{METHOD}

In this section we introduced the pipeline of Brain Atlas Individualization Network (BAI-Net), including spatial graph structure of the cortical surface, connectivity fingerprints of fiber-tract embedding, context-based node classification and its optimization.

\section{A. Spatial graph structure of the cortical surface}

Spatial graph structure of the individual brain graph was derived from the triangulated cortical surfaces (fs_LR32k surfaces) of each subject. These surfaces were extracted using the Freesurfer toolbox and Workbench commands[45, 46]. Each graph for each hemisphere consisted of around $30 \mathrm{k}$ vertices/nodes (removing the vertexes in the middle wall). The edges were constructed between each pair of two vertices that shared a triangle in the cortical surface. The brain graph was sparsely connected and highly localized in space, with each vertex connecting with 2-6 nearest vertices on average. The brain graph architecture provided a reference structure for each vertex to search for its spatial connectivity context.

\section{B. Connectivity fingerprints of fiber-tract embedding}

Many studies showed there exists a structure-function relationship and the identification of functional area through anatomical fingerprint $[28,29,47,48]$. In this study, the fibertract-based connectivity fingerprint was calculated, as the one of input in the graph convolutional network (shown in Fig. 1a):

1) A surface-based probabilistic tractography algorithm was used to track 5000 streamlines from each vertex on the cortical surface throughout the whole brain, including both cortical and subcortical regions [49-52]. The resulting streamline wholebrain tracking Track $_{s} \in \mathbb{R}^{N_{v} \times N_{b s}},\left(N_{v}\right.$ is the number of surface vertices and $N_{b s}$ is the numbers of brain voxels of subject $s$ ), had a threshold at 2 and then down-sampled to a $3 \mathrm{~mm}$ resolution to save computation time [53].

2) A individual-level binary mask for each of the whole-brain 72 fiber bundles $\operatorname{Tract}_{s} \in \mathbb{R}^{N_{b s} \times T}$ ( $T$ is the number of fiber tracts, names shown in Supplementary Table S1) was automatically segmented by a pre-trained deep-learning model named TractSeg [44]. And the resulting mask was also downsampled to a $3 \mathrm{~mm}$ resolution.

3) A fiber-tract-based embedding of the probabilistic tractography was conducted from the volume space $(50 \mathrm{k}+$ voxels) to the fiber-tract space (72 fiber bundles). The fingerprint $\mathcal{F} \in \mathbb{R}^{N_{v} \times T}$ is the set of feature vectors indicating the connectivity fingerprints $f_{i}$ defined on each vertex (here $N_{v}$ around 30k and $T=72$ ), shown below:

$$
\begin{gathered}
\mathcal{F}\left(f_{i, t}\right)=\frac{\sum_{k}^{N_{b s}} \operatorname{Track}_{s}(i, k) * \operatorname{Tract}_{s}(k, t)}{\sum_{k}^{N_{b s}} \operatorname{Tract}_{s}(k, t)} \\
\mathcal{F}\left(f_{i, t}\right)=\frac{f_{i, t}}{\sum_{t}^{T} f_{i, t}}
\end{gathered}
$$

where an element $f_{i, t}$ in the connectivity fingerprint was calculated by averaging the tracking connectivity from a vertex within a fiber tract mask. All fiber-tract elements were normalized to 1 for the fingerprint of a vertex.

Intuitively, this fingerprint means the averaged probability of anatomical fibers from a vertex to pass through each fiber tracts. Such fingerprints have biologically meaning, in the field of locating the similar functional brain areas across different nonhuman primate species[43].

\section{Context-based node classification}

Many previous parcellation algorithms treated each vertex or voxel independently while neglecting the context information of the neighborhoods by dividing the whole cortex into nonoverlapping regions [54]. Some researchers have attempted to utilize such context information for brain parcellations. For instance, Cohen and his colleagues attempted to detect local changes in functional connectivity maps through an edge detection algorithm on the cortical surface [55]. However, only the local context from the first-order neighborhood (directly connected vertices) was considered in their method. Graph convolutional networks provide a more generalized approach for integrating the context information at each node. One type 

available under aCC-BY-NC-ND 4.0 International license.

of graph convolution of $x$ using Chebyshev polynomials is defined as:

$$
g_{\theta} * x=\sum_{k=0}^{K} \theta_{k} T_{k}(L) x
$$

where $\tilde{L}$ is a normalized version of the graph Laplacian and is equal to $2 L / \lambda_{\max }-I$, with $\lambda$ being the largest eigenvalue. $\theta_{k}$ is the parameter to be learned for each order of the Chebyshev polynomial $T_{k},\left(T_{k}(x)=2 T_{k-1}(x)-T_{k-2}(x), T_{0}(x)=1\right.$, $T_{1}(x)=x$ ). By using a truncated expansion of the Chebyshev polynomial (as shown in Eq.1), the ChebNet graph convolution is naturally K-localized in the graph [56]. Specifically, when $K=1$, the graph convolution only considers the context information from the direct neighbors at each node. When $K>1$, the graph convolution also takes into account the information from a larger scale neighborhood, including nodes that can be reached within $\mathrm{K}$ steps of random walk on the graph. All this information is then integrated using the graph convolution. Two graph convolutional layers were stacked to enlarge the receptive fields of information integration. The GCN model took individual brain graphs as inputs, integrated the context information of brain connectivity at each vertex, and generated new representations (shown in Fig. 1 b).

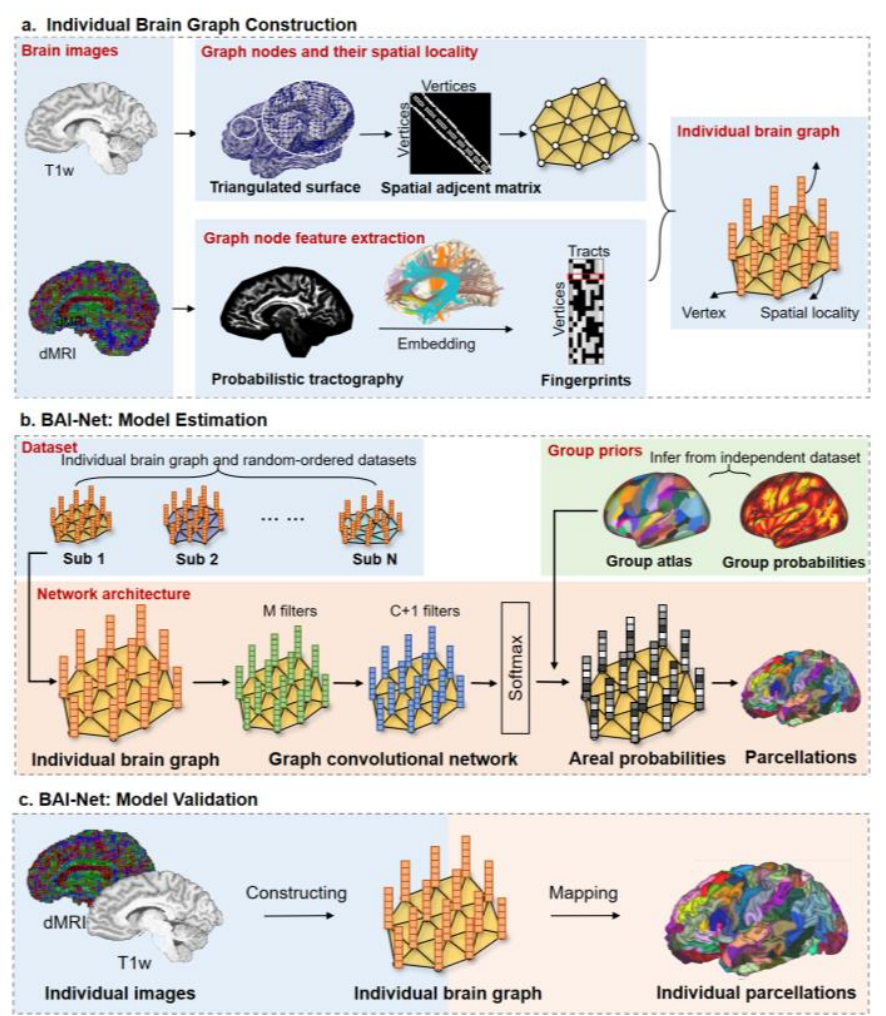

Fig. 1. Schematic diagram of the Brain Atlas Individualization Network (BAINet) using group priors. a: Construction of individual brain graph. b: BAI-Net: Model Estimation. c: BAI-Net: Model Validation.

\section{Optimization of BAl-Net Method}

The constructed individual brain graph dataset was used to train a GCN model with a group prior constraint (Fig. 1 b). (In this experiment, the group prior was extracted from 210 areas on the cortical surface of the Human Brainnetome Atlas), defined based on a group of 40 healthy subjects. The group atlas was first registered from the "fsaverage" surface template to individual surface space [57]. In addition to traditional dataset, A random-ordered dataset was created by randomly assigning the connectivity fingerprints to different nodes on the graph which introduced the random state of connectivity fingerprint in a local spatial region. In this situation, the label of whole brain surface was assigned with null label. This null model was used to train the robustness of the BAI-Net model when facing abnormal inputs such as distortions in brain anatomy. There were $C+l$ labels in a training model ( $C$ cortical areas plus a null label per hemisphere).

A one-hot encoding was generated at each vertex to indicate the labels of the cortical areas in the group atlas. This one-hot encoding vector was then used to modify the loss function for the GCN. Specifically, the GCN model takes an individual brain graph $\mathcal{G}=(\mathcal{V}, \mathcal{E}, \mathcal{F})$ as inputs, where $\mathcal{V}$ is the set of vertices (around $30 \mathrm{k}$ per hemisphere) in the cortical surface, $\mathcal{E}$ is the set of edges, showing whether two vertices share a common triangle in the surface, and $\mathcal{F} \in \mathbb{R}^{N_{v} \times T}$ is the set of feature vectors indicating the connectivity fingerprints $f_{i}$ defined on each vertex, $N_{v}$ is the number of vertices, $T$ is the number of fiber tracts (here $N_{v}$ around $30 \mathrm{k}$ and $T=72$ ). Using the validation dataset, optimal parameters for the two-layer graph model is chosen. The learned graph representations extracted from the last graph convolutional layer were then projected to a $(\mathrm{C}+1)$-dimensional probability vector at each vertex using the SoftMax function. The K-L divergence was used to calculated the difference between the group prior $y_{i, k}$ (one-hot encoding) and the predicted areal probability $p_{i, k}$ at each vertex $v_{i}$ for each region $k$. The weight of uncertainty $w_{i}$ at each vertex was inferred from the populational probability map of the group atlas. Thus, the final loss function was defined as follows:

$$
\text { Loss }=\sum_{i=1}^{N} w_{i} \sum_{k=0}^{K} y_{i, k} \log \left(p_{i, k}\right)
$$

The benefits of using the above loss function include: 1) High contributions of the vertices near the center of regions help to obtain a high level of inter-subject alignment for the global topographic organization. 2) Small contributions of the vertices at the borders of regions help retain the inter-subject variability to some degree and allow the mismatching of label assignments in the local architecture.

\section{DATASETS AND PREPROCESSING}

\section{HCP dataset}

We used healthy adult datasets from the S1200 Human Connectome Project (HCP) dataset, consisting of T1 weighted (T1w) data, resting-state functional MRI (rs-fMRI), as well as diffusion MRI (dMRI) data for each subject. Specifically, the BAI-Net model was trained and evaluated on the first dataset, consisting of 170 subjects (Age: 21-35) acquired from the Human Connectome Project S1200 release. The test-retest reliability of the model was then evaluated on the second dataset, consisting of 44 subjects acquired from the HCP test-retest datasets. And 969 subjects with three MRI modalities (removed subjects with larger fMRI head movements) were used for the predictions of individual cognition and behaviors. The dataset had been processed firstly by the HCP minimal preprocessing 

available under aCC-BY-NC-ND 4.0 International license.

pipeline $[45,46,58]$, and the cortical surfaces of a subject were reconstructed from T1-weighted MRI data and mapped onto the standard surface template with $32 \mathrm{k}$ vertices per hemisphere. Diffusion MRI data had been mainly preprocessed by motion correction, eddy current distortion correction, and echo-planar images (EPI) susceptibility-induced field distortion correction. The preprocessing of the functional MRI data mainly included motion correction, EPI susceptibility-induced distortion correction, linear trend removal, and independent component analysis (ICA)-based artifact removal. Further preprocessing details can be found in the HCP preprocessing pipeline [59, 60] (https://github.com/Washington-University/HCPpipelines ).

In further analysis, the HCP subject pairs were divided for four categories, 1) Unrelated: do not share any parent IDs; 2) Sibling: share one of parent IDs and not twins. 3) DZ: dizygotic twins in terms of the genetic records. 4) MZ: monozygotic twins in terms of the genetic records.

\section{MASiVar dataset}

An additional dataset from the Multisite, Multiscanner, and Multisubject Acquisitions for Studying Variability (MASiVar) dataset [61], consisting of 74 scans ( 8 scans were removed due to incomplete brain tissue data in the diffusion images) from 14 adult subjects ( 8 males and 6 females, age 27-47), in order to evaluate the impacts of multiple sessions, sites, and scanners. The dMRIs of these subjects were acquired from 3 cohorts using four scanners (two 3T Philips Achieva scanners used at two sites, one 3T General Electric Discovery MR750 scanner, and one 3T Siemens Skyra scanner) with at least one T1weighted image for each subject at each session. Of the three cohorts, different scanning sequences were used in diffusion imaging, including different $b$-values $\left(b=1000\right.$ to $\left.3000 \mathrm{~s} / \mathrm{mm}^{2}\right)$ and diffusion directions (about 40 96 directions for each $b$ value), different spatial resolutions ranging from $2.5 \mathrm{~mm}$ isotropic or $2.1 \mathrm{~mm}$ by $2.1 \mathrm{~mm}$ by $2.2 \mathrm{~mm}$ (sagittal, coronal, and axial), and so on. These diffusion images were preprocessed using the PreQual pipeline [62] with the default settings, including intensity normalization and distortion correction, as well as the Marchenko-Pastur technique[63-65]. More information on PreQual can be found at https://github.com/MASILab/PreQual.

\section{Statistical ANALYSis}

\section{A. Group-registered and iterative clustering methods}

Two individualized methods are compared in this study. One is group-registered method, that obtains individual parcellations based on group atlas in surface space. Groupregistered method maintains that the gyrus and sulcus of cortical areas are matched across subjects, which is also commonly used for atlas-based medical analysis in many studies. The group atlas on cortical surface is derived from mapping the group atlas in MNI space into the corresponding original templet, and then down-sampling into fsaverage LR32k surface space in which surface vertices are corresponded across subjects in terms of cortical gyrus and sulcus. For each subject, group registered method is mapping the fsaverage LR32k surface labels of group atlas into corresponding vertices of a subject's fsaverage LR32k surface.

Another is iterative clustering method, it iteratively updates global anatomical connectivity, and matches them with vertexlevel anatomical connectivity to identify individual cortical areas. This method was originally applied on image volume space [30], we adjusted it on individual cortical surface space (details of the method can be seen in Supplementary Methods).

\section{B. Robustness and specificity}

The robustness and specificity of individual parcellations were evaluated by the areal overlaps (Dice coefficient) between intra-subject and inter-subject pairs, using HCP test-retest dataset as well as from multi-scanner sessions using MASiVar dataset. In the calculation of areal overlaps, the surface parcellation for each subject was first converted into binary ROIs (C cortical areas in each hemisphere, one for each area), and were concatenated into a single vector. The Dice coefficient is calculated with the equation $(2 * \mathrm{~A} \cap \mathrm{B}) /(\mathrm{A}+\mathrm{B})$ between two vectors [13] for any area or parcellation overlaps mentioned in this article. All cortical surfaces were created using the HCP workbench.

\section{Inter-subject variability}

Inter-subject variability of a property is estimated by the effect size, Cohen's $d$, which reveals the real inter-subject variations after removing the intra-subject variations. Thus, inter-subject variability (the effect size) was defined as:

$$
V_{\text {inter }}=\frac{\mu_{\text {inter }}-\mu_{\text {intra }}}{\sqrt{\sigma_{\text {inter }}^{2}+\sigma_{\text {intra }}^{2}}}
$$

Where $\mu_{\text {inter }}$ and $\sigma_{\text {inter }}$ represent the mean and standard deviation of the variabilities between each pair of subjects, while $\mu_{\text {intra }}$ and $\sigma_{\text {intra }}$ represent those in the variabilities between different scans for the same subject.

We assumed that the individualized parcellation maps generated by the BAI-Net model would be able to retain the individual variability in brain morphology and connectivity. To test this hypothesis, we used different variability measures for brain parcellation (areal topography), brain morphology (modulated surface area), and brain connectivity (anatomical connectivity fingerprint). Modulated surface area was calculated by the averaged vertex area within a cortical region ('surface-vertex-areas' command in workbench software). The variability in brain morphology was measured by the absolute difference in the modulated surface area of the same cortical region between two parcellations. The variability in anatomical fingerprint was calculated by the absolute difference in the mean areal fingerprint between two parcellations. Additional association analysis was performed between the inter-subject variability in parcellation and brain morphology and brain connectivity.

\section{Population scores on the location and connectivity}

We investigated whether the group characteristics of individual parcellations were improved in the aspect of locations and connectivity. The scores were calculated with below definitions: 1) The similarity of maximum probability map: the maximum probability maps (MPM) were annotated as the area with the highest probability at each vertex. The similarity is calculated using areal overlaps between the MPM of individual parcellations and the group atlas. 2)Inter-subject 

available under aCC-BY-NC-ND 4.0 International license.

similarity of connectivity fingerprint: the connectivity fingerprint similarity of a cortical area was first calculated by the averaged Pearson correlation coefficient of areal connectivity fingerprint across subjects. Then the similarity of all cortical areas was averaged, weighted by the vertex number of each area. We tested the significance of population scores between two methods using the bootstrap technique (1000 samplings, 30 subjects per sampling).

E. Individual cognition and behavior predictions based on individual-specific topography

The topography of individual parcellation could reflect the individual cognition and behaviors [37]. To test the cognition and behavior associations, we adopted the kernel ridge regression (KRR), which combines ridge regression (linear least squares with L2-norm regularization) and kernel measurements. In here, we used the Dice coefficient as the kernel function $k(\cdot)$ and regularization parameter $\alpha=1$. The model can be described as below equations:

$$
C_{s}=\sum_{i=0}^{N_{\text {train }}} k\left(p_{s}, p_{i}\right) w_{i}=D_{s} W,
$$

the $D_{S} \in \mathbb{R}^{N_{\text {train }}}$ was the topographical similarity (Dice kernel) between the selected parcellation $p_{s}$ with the training parcellations $p_{i}, i=1, \ldots, N_{\text {train }}$. The $C_{s}$ and $C_{s}$ were the original and predicted cognition behavior scores of subject $s$, respectively. $W$ was the vector of regression parameters for training subjects. The optimal parameters $W$ were obtained by the minimization of below equation (Eq.7):

$$
\min _{w}\|D W-\mathrm{C}\|_{2}^{2}+\alpha\|W\|_{2}^{2} .
$$

For each individual cognition and behavior, the model was implemented in ten-fold cross validation loops for ten times. Any training sample whose parent overlapped with testing samples was removed first. The accuracy in each time was calculated by the Pearson correlation between all the predictions and observations. The model performance was evaluated by the mean accuracy in ten trails.

\section{F. The heritability of individual-specific topography}

The topography of individual functional networks can be explained proportionally by the genetic variation among individual in a population [66]. The genetic effect of the topography should also be revealed in the region-level cerebral cartography. So, we calculated the heritability of individualspecific topography according to the scripts on the websites (https://github.com/kevmanderson/h2_multi/blob/master/h2 m ulti/h2 multi.m). A multivariate linear mixed effects model was built as follows:

$$
Y=X B+G+E
$$

where $Y$ was the multi-dimension phenotype, $X$ was the covariates (including age, sex, age ${ }^{2}$, sex $\times$ age, age ${ }^{2} \times$ sex, total surface area and FreeSurfer-derived intracranial volume.), $B$ was the fixed effects, $G$ was the additive genetic effects from single nucleotide polymorphism (SNP) and $E$ was the unique environmental factors. The detailed calculation of the heritability and its significance can be seen in [66].

\section{EXPERIMENTAL RESULTS}

\section{A. Graph model training setting}

We used the population priors from Human Brainnetome Atlas [14] in the experiment. The detail utilization of datasets was shown in Supplementary Fig. S1. Through the validation testing, the model super-parameters were optimized through the grid research to achieve a better performance but with lower model parameters to avoid overfitting. A 5th-order graph convolution was used in GCN with $M=32$ convolutional kernels in the first GCN layer and $C+1=106$ kernels in the second GCN layer. We used Adam as the optimizer with an initial learning rate of 0.01 (decreased to 0.0001 after the 5th epoch). An additional L2 regularization of 0.0005 on weights was used to control model overfitting and noise in the imaging data. The network was trained on 100 subjects for 50 epochs with the batch size set to 1 (processing one subject at a time) using traditional dataset and random-order dataset respectively and evaluated on the validation set of 20 subjects at the end of each training epoch. The best model over 50 training epochs, that is, the one that achieved the lowest loss on the validation set, was saved and further evaluated on the independent test set of 50 subjects (Supplementary Fig. S2). During the model evaluation (Fig. 1c), an individual brain graph was first constructed from the test subject, using surface construction, diffusion tractography, and fiber-tract embedding. Then, the GCN model took the brain graph constructed from the target subject as input and predicted the areal probability vector at each vertex. Next, each vertex was assigned to the one of the 105 cortical areas for which it showed the highest probability. The predicted labels from both hemispheres were then combined to generate the final individualized brain atlas. The graph model is trained with Tensorflow framework using the 12G GeForce GTX 1080K.

\section{$B$. The high robustness and specificity of individual-} specific topography

The robustness of individual-specific topography was first evaluated using the 44 healthy subjects from HCP test-retest datasets. The topography overlaps between intra- and intersubject pairs of the BAI-Net individualized parcellation maps were shown in Fig. 2a. The result illustrated that highly reproducible parcellation maps were generated at the subject level (Dice $=0.902 \pm 0.012$ for whole cerebral cortex). At the meantime, the individual topography also showed a much high variability between subjects (Dice $=0.729 \pm 0.027$ for whole cerebral cortex), which was significant $\left(p\right.$ values $<10^{-50}$ ) to the intra-subject results. Examples of BAI-Net individualized parcellation maps in the right hemisphere are shown in Fig. $2 \boldsymbol{b}$ (maps of the left hemisphere shown in Supplementary Fig. S3). The BAI-Net model illustrated that detected variations in the topography (e.g., A39rv and A45c cortical areas) are large across subjects but maintain the specificity of a subject. The BAI-Net method shown a higher topography gap (0.17) between intra- and inter-subject pairs than IC method (0.12) on average (The topography overlaps between intra- and intersubject pairs for the IC method is $0.916 \pm 0.009$ and $0.800 \pm 0.015$ ). 

available under aCC-BY-NC-ND 4.0 International license.

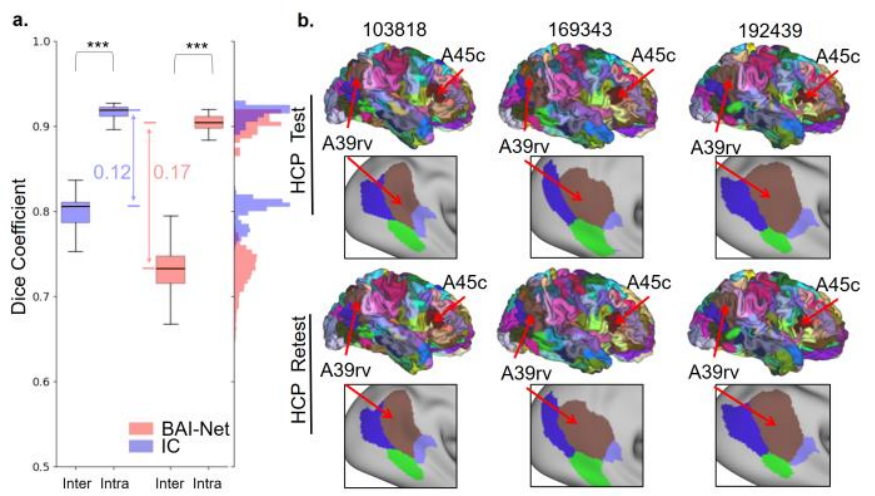

Figure 2. Reproducibility and specificity of individual topography produced by BAI-Net model. a: intra- and inter-subject overlaps between parcellations using HCP test-retest dataset for IC and BAI-Net methods. The *** indicates the significance $(p<0.001)$ by two-sample $t$ test. $b$ : the individual topography of three BAI-Net parcellations using the test-retest dataset.

The generalizability of the individualized parcellations derived from the BAI-Net model (pre-trained on HCP testing dataset) was further evaluated by directly applied on the MASiVar dataset. As shown in Fig. $3 \boldsymbol{a}$, the BAI-Net parcellation yielded much higher variability between different subjects than within the same subject, not only when using the same scanners (Dice score $=0.860 \pm 0.024$ and $0.707 \pm 0.027$, respectively for intra-subject and inter-subject pairs), but also when using different scanners (Dice score $=0.830 \pm 0.015$ and $0.703 \pm 0.025$, respectively for intra-subject and inter-subject pairs). Four examples from four scanners were highly similar and shown in Fig. 3b. It is worth noting that both the intra- and inter-subject overlaps were slightly lower in the multi-scanner dataset (MASiVar) than those in the single-scanner HCP dataset. The BAI-Net method had a degradation on the model performance (the same to the IC method, Fig. $3 a$ ), while BAINet maintained such topography variability across different scanners. Specifically, we qualified the inter-subject topography variability of whole cortex by effect size (Cohen's d). The BAI-Net method showed similar performance for the same scanner (Cohen's $d=4.23$ ) and different scanners (Cohen's $d=4.35$ ) while for the IC method, it decreased from 6.64 into 3.31 .

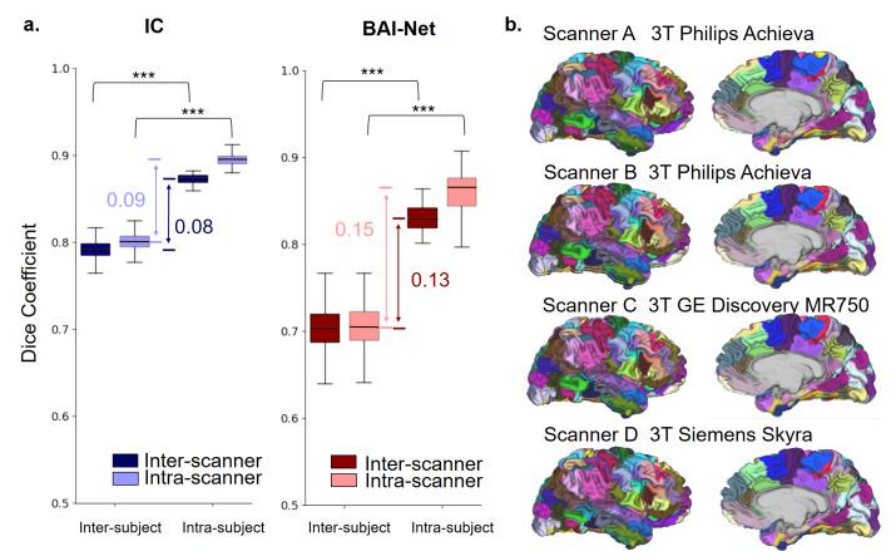

Figure 3. Generalizability of the individualized parcellations. a) intra- and intersubject parcellation overlaps on multiple scanners using the MASiVar dataset for IC and BAI-Net methods. The $* * *$ indicats the significance $(\mathrm{p}<0.001)$ by two-sample $t$ test. b) Four examples of BAI-Net parcellations on the subject 'sub-cIIs04' on different sites with four scanners.

Above all, the BAI-Net model captured reliable individual- specific signatures on various scopes. Large inter-subject variations were detected in the parcellations of different subjects. Our results demonstrated that the BAI-Net model generalized well on large populations either acquired from one single scanner or using multiple scanners.

\section{Inter-subject topography variability was associated with both brain connectome and morphology}

Besides the robustness, specificity, we also quantified the area distribution of the inter-subject topography variability (Cohen's $d$ ). And we investigated the correlations between the inter-subject topography variability with the inter-subject variability of areal connectivity and morphology.

As shown in Fig. 4, the BAI-Net individualized parcellation maps showed a large effect size of inter-subject topography variability at the whole-brain level. The topographic pattern of the effect size in the BAI-Net parcellations followed the functional and connectional gradient of the cortical organization [17]. Generally speaking, relatively small intersubject variations were observed in the primary cortex, e.g., the primary motor and sensory cortex. Higher effect sizes were detected in the association cortex, especially for brain regions that are involved in higher-order cognitive functions, e.g., the middle frontal gyrus (MFG) and inferior parietal lobule (IPL), in which also exhibited greater functional variability [17].

Moreover, we found a strong association in the variability maps between the brain parcellation and the connectivity fingerprints $(r=0.51, p<10-14)$. By contrast, a significant association with brain morphology was only detected for regions with relatively low variability in the brain parcellation (effect size $<2.3, \mathrm{r}=0.65, \mathrm{p}<10-13$ ) but not for highly variable brain regions (effect size $\geqslant 2.3, r=0.17, p=.08$ ). Our results indicated that the heterogeneity of the cortical organization revealed by the individualized parcellation maps was highly attributable to individual differences in brain connectivity rather than solely relying on variations in brain morphology.

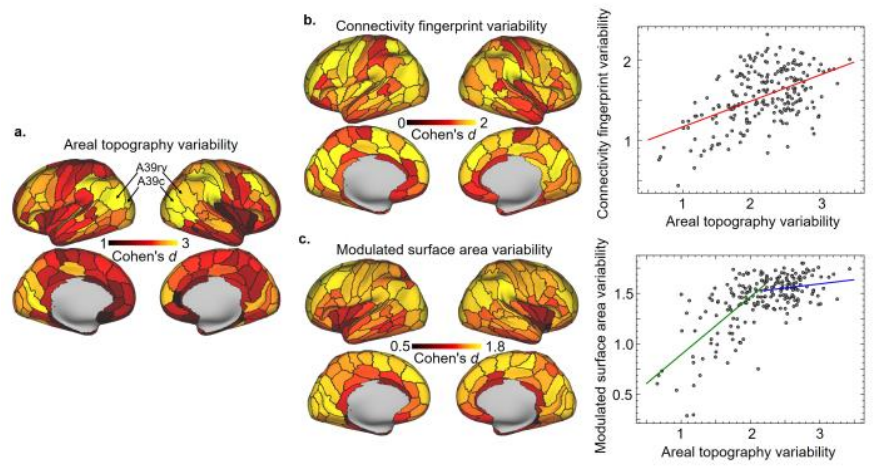

Figure 4. Inter-subject variability of individualized parcellations using the HCP test-retest dataset. Inter-subject areal variability for areal topography (a) are estimated by the effect size (Cohen's d score), as well as for connectivity fingerprint (b) and modulated surface area (c). The regression models were fit the relationship between areal topography and the other two features. Each dot in the right plot indicated one cortical area in the parcellation map.

\section{$D$. Higher group consistency in areal location and} anatomical connectivity.

Despite the variability of individual-specific topography coincided with the functional gradient of cerebral cortex on population, one would expect that the improvement in group characteristics of these individual populations. So, we 
bioRxiv preprint doi: https://doi.org/10.1101/2021.07.15.452577; this version posted November 21,2021 . The copyright holder for this preprint (which was not certified by peer review) is the author/funder, who has granted bioRxiv a license to display the preprint in perpetuity. It is made available under aCC-BY-NC-ND 4.0 International license.

calculated and compared two population scores for two methods. From the location aspects (Fig. 5a), the BAI-Net method showed a higher score on the similarity of maximum probability map than IC method on average (BAI-Net: 0.880; IC: 0.855 ; two-sample $t$ test, $\mathrm{p}<0.001$ ), indicating a better group consistent in area spatial distribution. The BAI-Net have constrained effectively individual cortical areas using the group priors to avoid population deviations. From connectivity aspects $(\boldsymbol{F i g} . \mathbf{5 b})$, the inter-subject similarity of connectivity fingerprint was also higher using the BAI-Net method than the IC method on average (BAI-Net:0.970; IC: 0.957; two-sample $\mathrm{t}$ test, $\mathrm{p}<0.001)$. The improvement in the connectivity consistency gives a substantial basis in the cross-subject analysis for the individual-specific topography. Additional of A39rv examples of the improvement in the functional connectivity could also support the improvement of intersubject connectivity similarity (Supplementary Fig. S5). In total, our results indicated that the BAI-Net individualized parcellation retained the global organization of cerebral cortex that inherited from the group atlas, and also improved the intrinsic connectivity similarity across subjects.
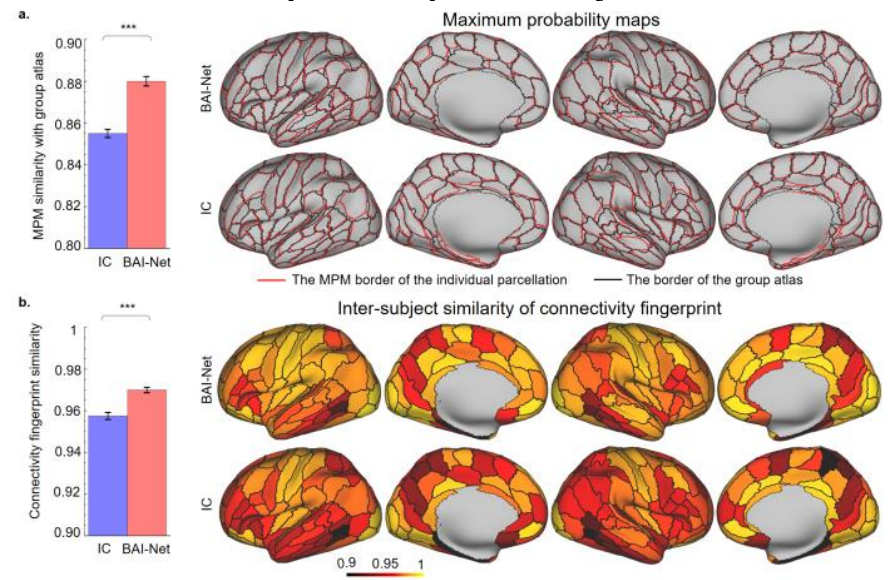

ectivity fingerprint

Figure 5. Population scores for IC and BAI-Net methods using HCP test dataset. a: the group similarity of maximum probability map and the MPM border of individual parcellations. b: inter-subject similarity of anatomical fingerprint for two methods. The significance of method comparisons was evaluated by using the bootstrap sampling of 30 subjects with 1000 times.

E. Individual-specific topography reflects better individual cognitive, behaviors and genetic associations

With the improvement in area alignment, the variations of individual-specific topography should reflect more differences of individual cognition and behaviors on a larger population. To test this, we applied a kernel ridge regression $[33,37]$ to predict individual cognition behaviors only based on areal topography of individual parcellations from IC or BAI-Net methods, respectively. As shown in Figure 6, we evaluated the performance on 58 cognition and behaviors. There are 31 cognition and behaves significantly predicted (at least in one trial $p<0.001$ ) by at least one of areal topographies in two individualized methods. The mean accuracy of 58 behaviors were $r=0.103$ and 0.075 for BAI-Net and IC methods, respectively. And the mean accuracy of 31 significant predicted behaviors were $r=0.155$ and 0.122 for BAI-Net and IC methods, respectively. So areal topography obtained from BAINet method showed higher associations with individual cognition and behaviors.

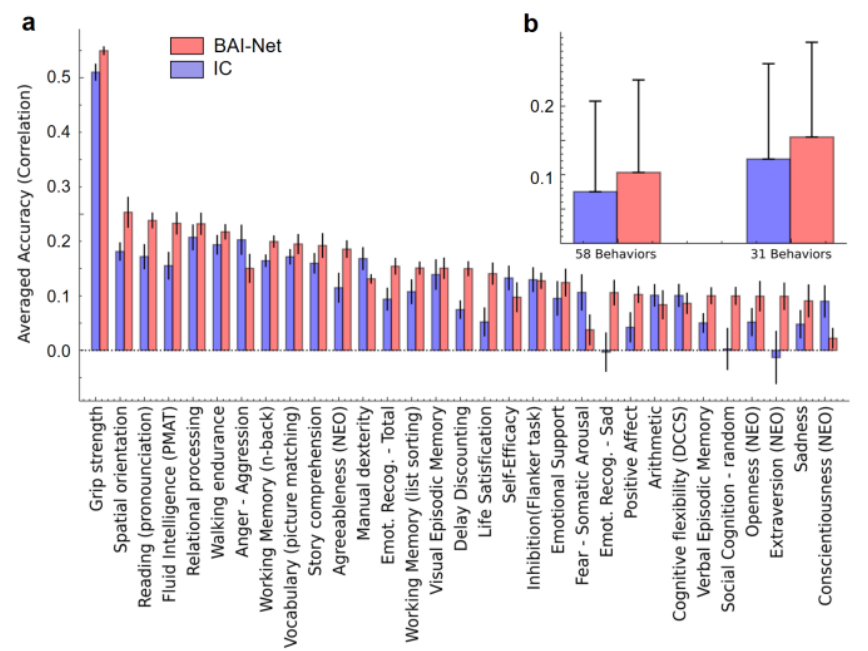

Figure 6. The prediction accuracy (correlation) of 58 individual cognition and behaviors using individual topography similarity in the ten-fold cross validation. We selected 31 significant cognition and behaviors that are significantly predicted $(\mathrm{p}<0.001)$ by at least one of methods. a: the averaged performance on total cognition and behaviors as well as 31 significant ones. b: the detailed performance on 31 significant cognition and behaviors.

We also estimated the individual-specific topography in genetic aspect. First, we analyzed the overlaps of individualspecific topography for four groups: Unrelated, Sibling, MZ and DZ (Figure 7). The results showed that the topography overlaps of BAI-Net parcellations were increasing with more closed kinship. For BAI-Net method, the overlaps between MZ pairs (Dice: 0.783 ) were higher than the Unrelated pairs (Dice: 0.733). However, such kinship reflection in IC method was relatively weak. The overlap difference between MZ and unrelated subject pairs $(0.013)$ was less than this in BAI-Net method (0.050). Moreover, based on the genetic analysis, the heritability of topography using the BAI-Net method $\left(\mathrm{h}^{2}=0.18\right.$, $\mathrm{p}<0.001)$ was much higher than that using IC method $\left(\mathrm{h}^{2}=0.09\right.$, $\mathrm{p}<0.001)$. And the extent of heritability for BAI-Net individualspecific parcellations was coincided with the individualspecific functional networks [66].

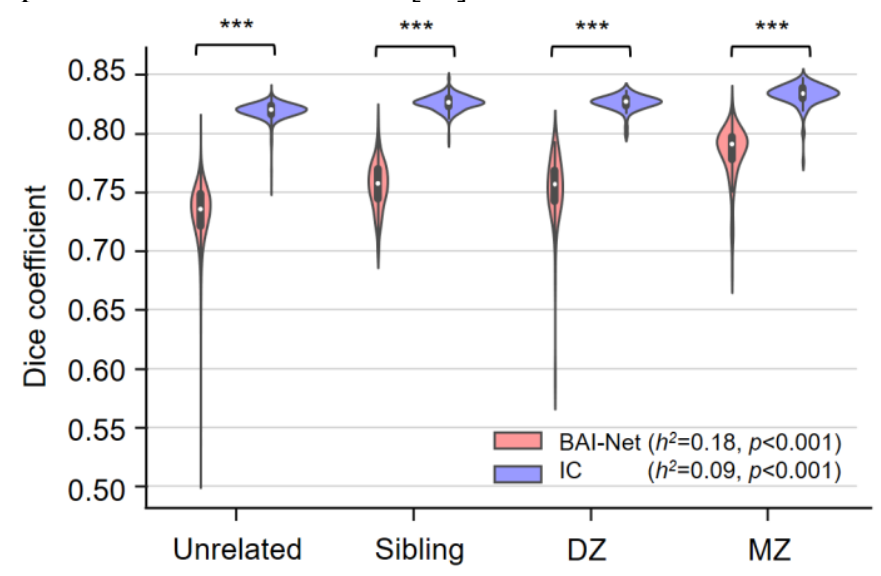

Figure 7. Similarity in the topographical organization of individual brain parcellations on four different sets, i.e., unrelated, sibling, DZ and MZ. We found the IC method achieved higher similarity among all four groups while the BAI-Net method captured more variance in brain parcellation among unrelated pairs. The $* * *$ indicates the significance $(\mathrm{p}<0.001)$ by paired $t$ test. Moreover, the BAI-Net method showed higher heritability of individualized parcels $(\mathrm{h} 2=0.18, \mathrm{p}=0.001)$. 

available under aCC-BY-NC-ND 4.0 International license.

\section{DISCUSSION}

An individualized brain atlas is one of approaches to understanding individual behaviors, physiology, and associated brain diseases. We developed the brain atlas individualization network (BAI-Net) from an anatomical connectivity view to automatically parcellate the cerebral cortex into segregated areas. The BAI-Net integrates the explainable representation of anatomical fingerprints with the connection context extracted by graph convolution networks in irregular space. The presented method provided highly reproducible, individualspecific topography under a variety of acquisition environments. These individual-specific parcellations improved the crosssubject analysis in the topography-based prediction of individual cognition and behaviors.

Traditionally, individual parcellations were derived from group-registered method (e.g., sulcus-based registration on cortical surface) between individual $\mathrm{T} 1 \mathrm{w} / \mathrm{T} 2 \mathrm{w}$ images and a standard group atlas. Such methods (no matter on the imagelevel CNN [67] or surface-level GCN framework [68]) used the extrinsic geometry of the cerebral cortex as the features across subjects but neglect the intrinsic connectional architecture of an individual brain. Several studies indicated that there exist intersubject connectivity variabilities of human cerebral cortex. Individual-specific topography of rs-fMRI parcellations are first introduced and shown the significant predictions on individual cognitions, behaviors and mental illnesses, providing a lot of perspective and practical works for the clinics $[13,26$, $30,32,33,37,38,69]$. In this study, we prove that such association with individual cognitions, behaviors, not only exists in the individual-specific functional topography but also in the anatomical topography of an individual cerebral cortex.

These associations with individual cognitions, behaviors, might result from the improvement of the cross-subject alignment in the presented BAI-Net method. The first advantage of the BAI-Net method is the utilization of embedding connectivity fingerprints. It has the two advantages for cross-subject analysis: 1) Global-range biological distinction. Local anatomical features (e.g., fractional anisotropy, myelin maps) lack the ability to make such globalrange distinctions $[13,40]$, when they are applied to a distant brain region. In contrast, the fiber-tract-based fingerprint can identify all the areas together within a hemisphere, thus greatly reducing the number of classifiers. Moreover, such areal distinction based on the embedding fingerprints have crosssubject biological meaning [43, 47] and the patterns of model activation are conformed with anatomy structure of brain tissues (seen in Supplementary Fig. S6). 2) Individual-level ROIs. Previous methods also used image registration from a standard template to locate the fiber tract positions in individuals [43]. The BAI-Net method, which adopts a deeplearning-based tool (TractSeg), was able to locate 72 individual fiber tracts more accurately than registration methods. Moreover, ROI normalization for anatomical connectivity mitigates distance influence on dominated local connectivity in individual. Above all, these fingerprints are crucial for a more precise, well-defined anatomical reference basis for crosssubject analysis.

Moreover, the BAI-Net also shows the advantages in handling the relationships between the connectivity fingerprints and the cortical areas using group priors in below aspects: 1) Surface-based graph analysis. The fine-grained surface in the Human Connectome Project-style processing provides a more accurate spatial localization of the cortical areas. It is suitable to select the neighborhood of a vertex on the cortical surface [70]. 2) Connectivity context identification. The connectivity context provides additional information on the representation of a group area than the previous method. It enhances the reliability in the areal identification by taking the consideration of spatial connectivity information on surface. It enabled the reliable area identification and effectively improve the similarity of areal anatomical fingerprint across subjects.

The time consumption of the BAI-Net method to inference the cerebral cartography of a new subject is around $3 \mathrm{~h}$ on the Centos 6 Linux system, with a preprocessed individual cortex surface. And the model supported the regional parcellation. The experiment shown that parcellating in a small region still works and highly overlaps with the whole-surface parcellation. This mode could partially solve the time problem of probabilistic tracking when only regional areas are needed (Supplementary Fig. S7).

BAI-Net provides an applicable individualization framework for probabilistic group atlases with the assistance of graph neural networks. A reliable topological arrangement of the cerebral cortex in individuals provides a basis for precise individual brain cortical analysis. Obtaining such an individualized analysis can enable the localization of high confidence physiological biomarkers, the exploration of individual structural and functional relationships, and the utilization of practical applications, such as identifying individual cortical areas for population-significant analysis, locating the target areas for transcranial magnetic stimulation (TMS) and deep brain stimulation (DBS) therapies, and reducing functional impairment in neurosurgery.

However, the presented model only used the information from structure and diffusion MRI. It would be better to achieve a better individual parcellations when multi-modality images are involved. The Glasser et al. presented an example of multimodality method [13]. With more information involved, it could be better to achieve a better reflection of individual differences on cognition, behaviors, and even many mental diseases with high precision. Moreover, for a more precise clinical analysis across different scanners, the bias between different scanners could be harmonized in advance based on large image samples from different scanners.

\section{CONCLUSION}

The presented pipeline and its methodological rationale will be also useful for individualizing other atlases (Julich-Brain [71]) based on individual anatomical connectivity profiles rather than based on morphological registration from individual T1w images. The connectivity fingerprints and connectivity context derived from graph model, provide a more reliable and highly reproducible individual parcellations. Based on the individual parcellations as well as areal inter-subject variations, the functional connectomes could achieve a better association with many individual cognition behaviors on average than group-registered and iterative clustering methods. 

available under aCC-BY-NC-ND 4.0 International license.

\section{REFERENCES}

[1]. K. Brodmann., Vergleichende Lokalisationslehre der Großhirnrinde in ihren Prinzipien dargestellt auf Grund des Zellenbaues. leipzig Germany: von Johann Ambrosius Barth, 1909.

[2]. C. Vogt. and O. Vogt., Allgemeinere Ergebnisse unserer Hirnforschung. Journal für Psychologie und Neurologie, 1919. 25(Suppl. 1): p. 273-462.

[3]. D.L. Collins, P. Neelin, T.M. Peters and A.C. Evans, Automatic $3 D$ intersubject registration of $M R$ volumetric data in standardized Talairach space. J Comput Assist Tomogr, 1994. 18(2): p. 192-205.

[4]. K. Zilles and K. Amunts, Centenary of Brodmann\"s map - conception and fate. Nature Reviews Neuroscience, 2010. 11(2): p. 139-145.

[5]. M.J. Hawrylycz, et al., An anatomically comprehensive atlas of the adult human brain transcriptome. Nature, 2012. 489(7416): p. 391-399.

[6]. K. Amunts, et al., BigBrain: An Ultrahigh-Resolution 3D Human Brain Model. Science, 2013. 340(6139): p. 14721475.

[7]. K. Amunts, H. Mohlberg, S. Bludau and K. Zilles, JulichBrain: A $3 D$ probabilistic atlas of the human brain's cytoarchitecture. Science, 2020.

[8]. B.T. Yeo, et al., The organization of the human cerebral cortex estimated by intrinsic functional connectivity. J Neurophysiol, 2011. 106(3): p. 1125-65.

[9]. J.D. Power, et al., Functional Network Organization of the Human Brain. Neuron, 2011. 72(4): p. 665-678.

[10]. D.C. Van Essen, Cartography and Connectomes. Neuron, 2013. 80(3): p. 775-790.

[11]. X. Shen, F. Tokoglu, X. Papademetris and R.T. Constable, Groupwise whole-brain parcellation from resting-state fMRI data for network node identification. Neuroimage, 2013. 82: p. 403-415.

[12]. E.M. Gordon, T.O. Laumann, B. Adeyemo, J.F. Huckins and S.E. Petersen, Generation and Evaluation of a Cortical Area Parcellation from Resting-State Correlations. Cerebral Cortex, 2014. 26(1): p. 288-303.

[13]. M.F. Glasser, et al., A multi-modal parcellation of human cerebral cortex. Nature, 2016. 536(7615): p. 171-+.

[14]. L. Fan, et al., The Human Brainnetome Atlas: A New Brain Atlas Based on Connectional Architecture. Cereb Cortex, 2016. 26(8): p. 3508-26.

[15].X.N. Zuo and X.X. Xing, Test-retest reliabilities of resting-state FMRI measurements in human brain functional connectomics: A systems neuroscience perspective. Neuroscience and Biobehavioral Reviews, 2014. 45: p. 100-118.

[16]. A.W. Toga, P.M. Thompson, S. Mori, K. Amunts and K. Zilles, Towards multimodal atlases of the human brain. Nature Reviews Neuroscience, 2006. 7(12): p. 952-966.

[17]. S. Mueller, et al., Individual variability in functional connectivity architecture of the human brain. Neuron, 2013. 77(3): p. 586-95.

[18]. M.D. Fox, H.S. Liu and A. Pascual-Leone, Identification of reproducible individualized targets for treatment of depression with TMS based on intrinsic connectivity. Neuroimage, 2013. 66: p. 151-160.
[19]. M.D. Fox, et al., Resting-state networks link invasive and noninvasive brain stimulation across diverse psychiatric and neurological diseases. Proceedings of the National Academy of Sciences of the United States of America, 2014. 111(41): p. E4367-E4375.

[20].E.W. Dickie, et al., Personalized Intrinsic Network Topography Mapping and Functional Connectivity Deficits in Autism Spectrum Disorder. Biological Psychiatry, 2018. 84(4): p. 278-286.

[21]. R. Patriat, et al., Individualized tractography-based parcellation of the globus pallidus pars interna using $7 T$ MRI in movement disorder patients prior to DBS surgery. 2018. 178: p. 198-209.

[22]. E.M. Gordon, et al., Precision Functional Mapping of Individual Human Brains. Neuron, 2017. 95(4): p. 791$807 \mathrm{e} 7$.

[23]. A.J. Fisher, J.D. Medaglia and B.F. Jeronimus, Lack of group-to-individual generalizabilityis a threat to human subjects research. Proceedings of the National Academy of Sciences of the United States of America, 2018. 115(27): p. E6106-E6115.

[24]. L. Fan, Mapping the Human Brain: What is the Next Frontier? The Innovation, 2020. 2(1): p. 100073.

[25]. E.S. Finn, et al., Functional connectome fingerprinting: identifying individuals using patterns of brain connectivity. Nature Neuroscience, 2015. 18(11): p. 1664-1671.

[26]. D.H. Wang, et al., Parcellating cortical functional networks in individuals. Nature Neuroscience, 2015. 18(12): p. 1853-1860.

[27]. E.M. Gordon, et al., Generation and Evaluation of a Cortical Area Parcellation from Resting-State Correlations. Cereb Cortex, 2016. 26(1): p. 288-303.

[28]. D.E. Osher, et al., Structural Connectivity Fingerprints Predict Cortical Selectivity for Multiple Visual Categories across Cortex. Cerebral Cortex, 2016. 26(4): p. 1668-1683.

[29]. R.E. Passingham, K.E. Stephan and R. Kotter, The anatomical basis of functional localization in the cortex. Nat Rev Neurosci, 2002. 3(8): p. 606-16.

[30]. M. Han, et al., Individualized Cortical Parcellation Based on Diffusion MRI Tractography. Cereb Cortex, 2020. 30(5): p. 3198-3208.

[31]. A. Schaefer, et al., Local-Global Parcellation of the Human Cerebral Cortex from Intrinsic Functional Connectivity MRI. Cerebral Cortex, 2018. 28(9): p. 30953114.

[32]. M. Salehi, A. Karbasi, X. Shen, D. Scheinost and R.T. Constable, An exemplar-based approach to individualized parcellation reveals the need for sex specific functional networks. Neuroimage, 2018. 170: p. 54-67.

[33]. R. Kong, et al., Spatial Topography of Individual-Specific Cortical Networks Predicts Human Cognition, Personality, and Emotion. Cereb Cortex, 2019. 29(6): p. 2533-2551.

[34]. M. Li, et al., Performing group-level functional image analyses based on homologous functional regions mapped in individuals. PLoS Biol, 2019. 17(3): p. e2007032.

[35]. E.M. Gordon, et al., Individual-specific features of brain systems identified with resting state functional correlations. Neuroimage, 2017. 146: p. 918-939. 

available under aCC-BY-NC-ND 4.0 International license.

[36]. M. Chong, et al., Individual parcellation of resting fMRI with a group functional connectivity prior. Neuroimage, 2017. 156: p. 87-100.

[37]. R. Kong, et al., Individual-Specific Areal-Level Parcellations Improve Functional Connectivity Prediction of Behavior. Cerebral Cortex, 2021. 00: p. 124.

[38].D.H. Wang, et al., Individual-specific functional connectivity markers track dimensional and categorical features of psychotic illness. Mol Psychiatry, 2018.

[39]. A. Henssen, et al., Cytoarchitecture and probability maps of the human medial orbitofrontal cortex. Cortex, 2016. 75: p. 87-112.

[40]. T. Ganepola, et al., Using diffusion MRI to discriminate areas of cortical grey matter. Neuroimage, 2018. 182: p. 456-468.

[41]. X.N. Zuo, T. Xu and M.P. Milham, Harnessing reliability for neuroscience research. Nature Human Behaviour, 2019. 3(8): p. 768-771.

[42]. D.M. Morris, K.V. Embleton and G.J. Parker, Probabilistic fibre tracking: differentiation of connections from chance events. Neuroimage, 2008. 42(4): p. 1329-39.

[43]. R.B. Mars, et al., Whole brain comparative anatomy using connectivity blueprints. Elife, 2018. 7.

[44]. J. Wasserthal, P. Neher and K.H. Maier-Hein, TractSeg Fast and accurate white matter tract segmentation. NeuroImage, 2018. 183: p. 239-253.

[45]. D.S. Marcus, et al., Informatics and data mining tools and strategies for the human connectome project. Front Neuroinform, 2011. 5: p. 4.

[46]. M.F. Glasser, et al., The minimal preprocessing pipelines for the Human Connectome Project. Neuroimage, 2013. 80: p. $105-24$.

[47]. R.B. Mars, et al., Comparing brains by matching connectivity profiles. Neurosci Biobehav Rev, 2016. 60: p. 90-7.

[48]. D. Wu, et al., Hierarchy of Connectivity-Function Relationship of the Human Cortex Revealed through Predicting Activity across Functional Domains. Cereb Cortex, 2020.

[49]. T.E.J. Behrens, H.J. Berg, S. Jbabdi, M.F.S. Rushworth and M.W. Woolrich, Probabilistic diffusion tractography with multiple fibre orientations: What can we gain? Neuroimage, 2007. 34(1): p. 144-155.

[50]. S. Jbabdi, S.N. Sotiropoulos, A.M. Savio, M. Grana and T.E.J. Behrens, Model-based analysis of multishell diffusion MR data for tractography: How to get over fitting problems. Magnetic Resonance in Medicine, 2012. 68(6): p. 1846-1855.

[51]. M. Hernandez, et al., Accelerating Fibre Orientation Estimation from Diffusion Weighted Magnetic Resonance Imaging Using GPUs. Plos One, 2013. 8(4).

[52]. M. Hernandez-Fernandez, et al., Using GPUs to accelerate computational diffusion MRI: From microstructure estimation to tractography and connectomes. Neuroimage, 2019. 188: p. 598-615.

[53].H. Li, et al., ATPP: A Pipeline for Automatic Tractography-Based Brain Parcellation. Frontiers in Neuroinformatics, 2017. 11.
[54]. S.B. Eickhoff, B.T.T. Yeo and S. Genon, Imaging-based parcellations of the human brain. Nat Rev Neurosci, 2018. 19(11): p. 672-686.

[55]. A.L. Cohen, et al., Defining functional areas in individual human brains using resting functional connectivity MRI. Neuroimage, 2008. 41(1): p. 45-57.

[56]. M. Defferrard, X. Bresson and P. Vandergheynst, Convolutional Neural Networks on Graphs with Fast Localized Spectral Filtering. Advances in Neural Information Processing Systems 29 (Nips 2016), 2016. 29.

[57]. B. Fischl, M.I. Sereno, R.B.H. Tootell and A.M. Dale, High-resolution intersubject averaging and a coordinate system for the cortical surface. Human Brain Mapping, 1999. 8(4): p. 272-284.

[58]. D.C. Van Essen, et al., The WU-Minn Human Connectome Project: An overview. Neuroimage, 2013. 80: p. 62-79.

[59]. S.M. Smith, et al., Advances in functional and structural MR image analysis and implementation as FSL. Neuroimage, 2004. 23: p. S208-S219.

[60]. M. Jenkinson, C.F. Beckmann, T.E. Behrens, M.W. Woolrich and S.M. Smith, Fsl. Neuroimage, 2012. 62(2): p. 782-790.

[61]. L.Y. Cai, et al., MASiVar: Multisite, multiscanner, and multisubject acquisitions for studying variability in diffusion weighted MRI. Magnetic Resonance in Medicine, 2021.

[62]. L.Y. Cai, et al., PreQual: An automated pipeline for integrated preprocessing and quality assurance of diffusion weighted MRI images. Magnetic Resonance in Medicine, 2021.

[63]. J. Veraart, et al., Denoising of diffusion MRI using random matrix theory. Neuroimage, 2016. 142: p. 384-396.

[64]. J. Veraart, E. Fieremans and D.S. Novikov, Diffusion MRI Noise Mapping Using Random Matrix Theory. Magnetic Resonance in Medicine, 2016. 76(5): p. 1582-1593.

[65]. L. Cordero-Grande, D. Christiaens, J. Hutter, A.N. Price and J.V. Hajnal, Complex diffusion-weighted image estimation via matrix recovery under general noise models. Neuroimage, 2019. 200: p. 391-404.

[66]. K.M. Anderson, et al., Heritability of individualized cortical network topography. Proceedings of the National Academy of Sciences of the United States of America, 2021. 118(9).

[67]. A. Iqbal, R. Khan and T. Karayannis, Developing a brain atlas through deep learning. Nature Machine Intelligence, 2019. 1(6): p. 277-287.

[68]. K. Gopinath, C. Desrosiers and H. Lombaert, Graph Convolutions on Spectral Embeddings for Cortical Surface Parcellation. Medical Image Analysis, 2019. 54: p. 297-305.

[69]. D.J. Greene, et al., Integrative and Network-Specific Connectivity of the Basal Ganglia and Thalamus Defined in Individuals. Neuron, 2020. 105(4): p. 742-+.

[70]. T.S. Coalson, D.C. Van Essen and M.F. Glasser, The impact of traditional neuroimaging methods on the spatial localization of cortical areas. Proc Natl Acad Sci, 2018. 115(27): p. E6356-E6365. 
bioRxiv preprint doi: https://doi.org/10.1101/2021.07.15.452577; this version posted November 21,2021 . The copyright holder for this preprint (which was not certified by peer review) is the author/funder, who has granted bioRxiv a license to display the preprint in perpetuity. It is made available under aCC-BY-NC-ND 4.0 International license.

[71]. K. Amunts, H. Mohlberg, S. Bludau and K. Zilles, JulichBrain: A $3 D$ probabilistic atlas of the human brain's cytoarchitecture. Science, 2020. 369(6506): p. 988-992. 